\title{
Utilisation directe des graines de coton décortiquées de variétés sans gossypol dans l'alimentation des poulets de chair
} T. Yo* en Côte-d'Ivoire

\begin{abstract}
YO (T.). Utilisation directe des graines de coton décortiquées de variétés sans gossypol dans l'alimentation des poulets de chair en Côte-d'Ivoire. Revue Élev. Méd. vét. Pays trop., 1991, 44 (3) : 355-360

L'utilisation de graines de coton décortiquées sans gossypol (GCDSG) comme source de protéines et d'énergie dans l'alimentation des poulets de chair a été testée en comparant quatre rations contenant 0,10 15 ou 20 p 100 de GCDSG. Il n'y a pas eu d'effet significatif sur le gain de poids vif des animaux à 14,28 et 42 jours d'âge. Sur l'ensemble de la période expérimentale (0-42 jours), le taux de GCDSG dans l'aliment n'a pas eu d'effet significatif sur la quantite ingérée. Cependant, le régime à $20 \mathrm{p} .100$ de GCDSG a donné un indice de consommation plus élevé que ceux en contenant 10 ou $15 \mathrm{p} .100$ Le taux de mortalité n'a pas été affecté, mais on observe une tendance à l'augmentation de la fréquence de malformations des pattes. Sur la base d'un prix théorique des GCDSG de 42,6 francs CFA $/ \mathrm{kg}$, l'incorporation dans la ration diminue le coût alimentaire et augmen. te la marge brute par animal pour les aliments contenant 10 ou 15 p. 100 de GCDSG. Celles-ci pourraient constituer une nouvelle ressource intéressante pour l'alimentation des volailles. Mots clés : Poulet de chair - Alimentation - Graine de coton - Efficience nutritionnelle - Gain de poids - Côte-d'Ivoire.
\end{abstract}

\section{INTRODUCTION}

L'utilisation du tourteau de coton comme source de protéines dans l'alimentation des volailles est très répandue et a fait l'objet de nombreuses études $(5,16,18,19,23)$. Des travaux récents ont montré qu'il est possible de remplacer, dans les régimes alimentaires pour volailles, le tourteau de soja par le tourteau de coton sans gossypol pourvu que lion corrige sa faible teneur en lysine $(3,13,20,24)$.

L'expérience menée avait pour objectif de tester l'effet de l'incorporation, dans les rations, de la graine décortiquée sans gossypol (GCDSG) sur l'ingéré alimentaire, l'évolution pondérale et les performances économiques des poulets de chair de souche industrielle.

Si la graine de coton est largement utilisée dans l'alimentation des ruminants $(4,14,25)$, il y a peu d'études concernant les monogastriques ; vraisemblablement en raison de l'effet néfaste du gossypol présent en quantité élevée dans les graines des variétés classiques. Les essais d'utilisation de ces graines ont généralement

1. Institut des Savanes (IDESSA), Département Élevage, BP 633, Bouaké, Côte-d'Ivoire.

* Avec la collaboration technique de Kouassi YA.

Reçu le 10.10.1990, accepté le 6.6.1991. donné des résultats peu favorables avec les monogas triques. Ainsi, pour les porcs $(6,17)$ et les rats $(6,7)$, l'incorporation dans les rations de graines crues, entières ou décortiquées, a entraîné une chute des performances de croissance et une importante mortalité. L'utilisation chez les poules pondeuses d'aliments contenant 15 à 20 p. 100 de graines de coton a eu pour effet la diminution de l'ingestion alimentaire et du poids vif des poules, et une chute du taux de ponte (9). Contrairement à ces résultats, obtenus avec des graines à haute teneur en gossypol, l'utilisation des graines de coton glandless, en remplacement du maïs et du tourteau de soja, n'a pas eu d'effets négatifs sur le gain de poids des porcs et a permis d'améliorer leur indice de consommation (15).

De par leur teneur en lipides et en matières azotées, les graines de coton des variétés sans gossypol pourraient constituer une matière première intéressante pour l'alimentation des monogastriques.

\section{MATÉRIEL ET MÉTHODE}

L'essai a été réalisé à l'Institut des Savanes de Bouaké (Côte-d'lvoire), du 5 juillet au 16 août 1990. Au cours de cette période, la température moyenne était de $23,8^{\circ} \mathrm{C}$ et le taux d'humidité moyen de 88,6 p. 100.

\section{Matériel}

Six cent quatorze poussins d'un jour, de souche Vedette, pesant en moyenne $42 \mathrm{~g}$, ont été répartis en 16 lots homogènes de 38-39 poussins et élevés dans des parquets de $6 \mathrm{~m}^{2}$ chacun d'un poulailler au sol semi-ouvert. La litière était constituée par des balles de riz. Des graines de coton glandless (variété GL7) récoltées en 1989 ont été acquises après égrenage, puis décortiquées pour séparer la coque, essentiellement cellulosique, de l'amande. Le produit ainsi obtenu a été séché au soleil. Un échantillon a été ensuite prélevé pour l'analyse bromatologique suivant les méthodes décrites par l'AOAC (2). L'analyse chimique a donné la composition suivante par rapport à la matière sèche $: 31,6$ p. 100 de protéines brutes, 38,1 p. 100 de matières grasses, 10,8 p. 100 de cellulose brute et 5,6 p. 100 de cendres. La teneur en énergie métabolisable vraie, estimée suivant l'équation de SIBBALD (22), est d'environ $4800 \mathrm{kcal} / \mathrm{kg}$ de MS. 
Quatres rations ont été formulées contenant 0 p. 100 (aliment témoin), 10, 15, 20 p. 100 de GCDSG. Pour qu'elles soient chimiquement isoénergétiques et isoazotées, l'incorporation progressive de GCDSG fut associée à une augmentation de la part du son de blé (25 francs CFA/kg) par substitution au maïs ( 55 francs CFA/kg) et à la farine de poisson (160 francs CFA/kg), sans recherche d'un optimum économique. Les teneurs en minéraux furent ajustées entre les traitements. En période de démarrage (0-28 jours), les aliments contenaient environ $3000 \mathrm{kcal}$ EM $/ \mathrm{kg}$ d'aliment et 22,2 p. 100 de protéines. En période de croissance (29-42 jours), le taux de protéines dans les rations a été ramené à 20 p. 100 . Des doses croissantes de lysine et de méthionine synthétiques ont été incorporées aux régimes contenant de la GCDSG pour en équilibrer les concentrations en acides aminés.

La composition des régimes alimentaires et leurs teneurs en nutriments, calculées suivant les données de l'INRA (11), sont indiquées aux tableaux I et II.

TABLEAU I Composition des aliments de démarrage (0-28 jours).

\begin{tabular}{|l|c|c|c|c|}
\hline \multicolumn{1}{|c|}{ Taux de GCDSG (p. 100) } & 0 & 10 & 15 & 20 \\
\hline Matières premières (p. 100) & & & & \\
Maïs & 45 & 39,4 & 34,3 & 29 \\
Farine de poisson * & 18,9 & 14,4 & 12 & 9,5 \\
Graine de coton & 0 & 10 & 15 & 20 \\
Remoulage de blé & 3,6 & 3,8 & 5,9 & 8,1 \\
Farine basse riz & 20 & 20 & 20 & 20 \\
Tourteau de coton & 10 & 10 & 10 & 10 \\
Huile de palme & 1,1 & 0 & 0 & 0 \\
Phosphate bicalcique & 0,0 & 0,5 & 0,8 & 1,1 \\
Carbonate de calcium & 0,7 & 1,1 & 1,2 & 1,4 \\
Sel de cuisine & 0,2 & 0,2 & 0,2 & 0,2 \\
Prémix * & 0,4 & 0,4 & 0,4 & 0,4 \\
Lysine & 0,0 & 0,08 & 0,12 & 0,17 \\
Méthionine & 0,07 & 0,10 & 0,11 & 0,13 \\
& & & & \\
Teneur en nutriments calculée ${ }^{\star *}$ & & & & \\
Énergie (kcal EM/kg) & 3000 & 2996 & 3005 & 3009 \\
Protéines (N x 6,25) (p. 100) & 22,2 & 22,2 & 22,2 & 22,2 \\
Lysine (p. 100) & 1,2 & 1,2 & 1,2 & 1,2 \\
Méthionine (p. 100) & 0,6 & 0,6 & 0,6 & 0,6 \\
Acides aminés soufrés (p. 100) & 0,9 & 0,9 & 0,9 & 0,9 \\
Calcium (p. 100) & 1,5 & 1,5 & 1,5 & 1,5 \\
Phosphore disponible (p. 100) & 0,6 & 0,6 & 0,6 & 0,6 \\
Cellulose (p. 100) & 3,6 & 4,5 & 5,1 & 5,7 \\
Matières grasses (p. 100) & 7,8 & 9,6 & 11 & 12,3 \\
Prix (F CFA/kg) & 70 & 67,5 & 66,2 & 65,6 \\
\hline
\end{tabular}

* La farine de poisson' utilisée contenait 21 p. cent de cendres, $57 p$. cent de protéines et $4 \mathrm{p}$. cent de matière grasse. Sa teneur en $E M$ a été estimée à $2800 \mathrm{kcal} / \mathrm{kg}$.

+* Composition du prémix (par kg) : vit. A : 3000000 Ul; vit. E : 2500 UI ; vit. B2 : $1000 \mathrm{mg}$; vit. D3: $1250000 \mathrm{UI}$; vit. K: $1111 \mathrm{mg}$; vit. B12:2500 mg. Contient aussi: sulfate de cuivre, sulfate de fer, acide nicotinique, iodate de calcium, oxyde de manganèse, oxyde de zinc, chlorure de chaline, sélénium.

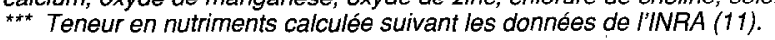

TABLEAU II Composition des aliments de croissance (29-42 jours).

\begin{tabular}{|l|c|c|c|c|}
\hline \multicolumn{1}{|c|}{ Taux de GCDSG (p. 100) } & 0 & 10 & 15 & 20 \\
\hline Matières premières (p. 100) & & & & \\
Maïs & 54 & 42,7 & 37 & 31,9 \\
Farine de poisson & 14,9 & 10 & 7,2 & 5 \\
Graine de coton & 0 & 10 & 15 & 20 \\
Remoulage de blé & 0 & 5 & 8 & 10 \\
Farine basse riz & 20 & 20 & 20 & 20 \\
Tourteau de coton & 10 & 10 & 10 & 10 \\
Phosphate bicalcique & 0,5 & 1,2 & 1,4 & 1,7 \\
Carbonate de calcium & 0 & 0,3 & 0,5 & 0,5 \\
Sel de cuisine & 0,2 & 0,3 & 0,3 & 0,3 \\
Prémix* & 0,4 & 0,4 & 0,4 & 0,4 \\
Lysine & 0 & 0,05 & 0,10 & 0,15 \\
Méthionine & 0,05 & 0,09 & 0,10 & 0,10 \\
& & & & \\
Teneur en nutriments calculée & & & & \\
Énergie (kcal EM/kg) & 3006 & 3003 & 3002 & 3014 \\
Protéines (N x 6,25) (p. 100) & 20,1 & 20,1 & 20,1 & 20,2 \\
Lysine (p. 100) & 1,0 & 1,0 & 1,0 & 1,0 \\
Méthionine (p. 100) & 0,5 & 0,5 & 0,5 & 0,5 \\
Acides aminés soufrés (p. 100) & 0,8 & 0,8 & 0,8 & 0,8 \\
Calcium (p. 100) & 1,1 & 1,1 & 1,1 & 1,0 \\
Phosphore disponible (p. 100) & 0,6 & 0,6 & 0,6 & 0,6 \\
Cellulose (p. 100) & 3,4 & 4,7 & 5,3 & 5,9 \\
Matières grasses (p. 100) & 6,7 & 9,4 & 10,7 & 12,0 \\
Prix (F CFA/kg) & 65,5 & 62,3 & 60,5 & 59,5 \\
\hline
\end{tabular}

* Composition du prémix (par kg) : vit. A: $3000000 \mathrm{Ul}$; vit. E : $2500 \mathrm{UI}$ vit $B 2: 1000 \mathrm{mg}$; vit. $D 3: 1250000 \mathrm{Ul}$; vit $\mathrm{K}: 1111 \mathrm{mg}$; vit. $\mathrm{B12}$ $2500 \mathrm{mg}$. Contient aussi : sulfate de cuivre, sulfate de fer, acide nicotinique iodate de calcium, oxyde de manganese, oxyde de zinc, chlorure de choline, sélénium.

** Teneur en nutriments calculée suivant les données de l'INRA (11).

Ces aliments ont été usinés sur la station, puis distribués aux poussins à raison de quatre parquets par traitement. Un dispositif en blocs a été adopté à l'intérieur du poulailler.

\section{Méthode}

Au cours des cinq premiers jours, un éclairement continu (lumière diurne + lumière artificielle nocturne) a été maintenu dans le poulailler. La durée totale d'éclairement a été réduite à $18 \mathrm{~h}$ par nycthémère en ramenant la durée de l'éclairement nocturne à $6 \mathrm{~h}$. Une température de 32$35{ }^{\circ} \mathrm{C}$ était maintenue dans les parquets pendant la première semaine à l'aide d'un chauffage électrique. L'eau et l'aliment étaient disponibles à volonté. Des vaccinations ont été faites contre la maladie de Newcastle et la bronchite infectieuse à 5 et 25 jours d'âge et contre la maladie de Gumboro à 12 et 28 jours d'âge. Un anticoccidien a été administré dans l'eau de boisson à 12 et 27 jours d'âge pendant quatre jours consécutifs. A 14,28 et 42 jours d'âge, les animaux ont été pesés individuellement. 
A ces dates, la consommation d'aliment correspondant aux deux semaines précédentes a été mesurée pour chaque parquet. De même, les morts et les animaux présentant une malformation des pattes ont été dénombrés.

La graine de coton entière est livrée par les usines d'égrenage du coton aux huileries et éleveurs au prix de 23 francs CFA $/ \mathrm{kg}$. On pourrait donc estimer le prix de la graine décortiquée à 42,6 francs CFA/kg, étant donné que le rendement au décorticage est en moyenne de 54 p. 100 (1). L'étude de la rentabilité économique des rations testées a été effectuée sur cette hypothèse de prix.

L'ensemble des données recueillies a été analysé à l'aide du programme STAT-ITCF en prenant en compte le facteur régime alimentaire $(R)$ pour l'analyse du poids vif et des gains de poids (modèle 1 ), le régime alimentaire $(R)$ et l'âge (A) pour l'analyse de l'ingéré alimentaire et l'indice de consommation (modèle 2 ). La prise en compte du facteur âge dans le modèle 2 permet de mesurer l'effet de l'interaction entre le régime alimentaire et l'âge des animaux sur l'ingéré alimentaire et l'indice de consommation.

Modèle $1: Y i k=u+R i+e i k$

Modèle $2: Y i j k=u+R i+A j+\left(R^{*} A\right) i j+e i j k$

$\mathrm{u}$ : moyenne générale ; Ri : effet de l'aliment $i ; A j$ : effet de l'âge j ; $\left(R^{*} A\right)$ ij : effet de l'interaction entre l'aliment $i$ et l'âge $\mathrm{j}$; eijk : variation résiduelle.

En cas d'effets significatifs des facteurs étudiés, les moyennes des traitements sont comparées à l'aide du "multiple range test" de Newman et Keuls.

Les taux de mortalité et d'animaux présentant des malformations de pattes ont été comparés à l'aide du test de $\mathrm{khi}^{2}$.

\section{RÉSULTATS}

\section{Performances zootechniques}

Les résultats relatifs aux performances pondérales, à l'ingéré alimentaire, à l'indice de consommation, aux taux de mortalité et de malformations des pattes sont résumés dans les tableaux III et IV.

L'incorporation de GCDSG dans les rations de démarrage et de croissance à des taux de 10 à 20 p. 100 n'a pas eu d'effet significatif sur le poids et le gain de poids à 14 , 28 ou 42 jours d'âge $(P>0,05)$. Le gain moyen quotidien (GMQ) augmente de 23 à $49 \mathrm{~g}$ entre le début et la fin de l'essai.

On observe un effet significatif de l'interaction entre l'âge et le régime alimentaire sur l'ingéré alimentaire et l'indice de consommation $(P<0,01)$. En effet, alors qu'au cours
TABLEAU III Performances pondérales, taux de mortalité et de malformation des pattes.

\begin{tabular}{|l|c|c|c|c|}
\hline \multicolumn{1}{|c|}{ Taux de GCDSG (p. 100) } & 0 & 10 & 15 & 20 \\
\hline Poids vif* & & & & \\
Poids départ (g) & 42 & 42 & 42 & 42 \\
Poids à 14 jours (g) & $348^{\mathrm{a}}$ & $362^{\mathrm{a}}$ & $367^{\mathrm{a}}$ & $357^{\mathrm{a}}$ \\
Poids à 28 jours (g) & $973^{\mathrm{a}}$ & $994^{\mathrm{a}}$ & $992^{\mathrm{a}}$ & $952^{\mathrm{a}}$ \\
Poids à 42 jours (g) & $1653^{\mathrm{a}}$ & $1677^{\mathrm{a}}$ & $1699^{\mathrm{a}}$ & $1618^{\mathrm{a}}$ \\
Taux de mortalité (p. 100) & $5,2^{\mathrm{a}}$ & $1,3^{\mathrm{a}}$ & $3,9^{\mathrm{a}}$ & $5,2^{\mathrm{a}}$ \\
Handicapés $^{\star}$ (p. 100) & $3,2^{\mathrm{a}}$ & $4,6^{\mathrm{a}}$ & $6,6^{\mathrm{a}}$ & $7,1^{\mathrm{a}}$ \\
\hline
\end{tabular}

* Les moyennes figurant sur la même ligne et portant la même lettre ne sont pas significativement différentes $(P>0,05)$.

** Poulets présentant une malformation quelconque des pattes.

TABLEAU IV Ingéré alimentaire et indice de consommation.

\begin{tabular}{|l|c|c|c|c|}
\hline \multicolumn{1}{|c|}{ Taux de GCDSG (p. 100) } & 0 & 10 & 15 & 20 \\
\hline Consommation d'aliments & & & & \\
$0-14$ jours $(\mathrm{g})$ & $491^{\mathrm{a}}$ & $525^{\mathrm{a}}$ & $507^{\mathrm{a}}$ & $480^{\mathrm{a}}$ \\
$15-28$ jours $(\mathrm{g})$ & $1638^{\mathrm{a}}$ & $1576^{\mathrm{a}}$ & $1448^{\mathrm{ab}}$ & $1350^{\mathrm{b}}$ \\
$29-42$ jours $(\mathrm{g})$ & $2074^{\mathrm{a}}$ & $2060^{\mathrm{a}}$ & $2176^{\mathrm{a}}$ & $2476^{\mathrm{b}}$ \\
$0-28$ jours $(\mathrm{g})$ & $2142^{\mathrm{a}}$ & $2101^{\mathrm{a}}$ & $1953^{\mathrm{a}}$ & $1827^{\mathrm{a}}$ \\
$0-42$ jours $(\mathrm{g})$ & $4216^{\mathrm{a}}$ & $4162^{\mathrm{a}}$ & $4129^{\mathrm{a}}$ & $4303^{\mathrm{a}}$ \\
& & & & \\
Indice de consommation & & & & \\
(g aliment/g gain de poids) & & & & \\
$0-14$ jours & $1,62^{\mathrm{a}}$ & $1,64^{\mathrm{a}}$ & $1,56^{\mathrm{a}}$ & $1,52^{\mathrm{a}}$ \\
$15-28$ jours & $2,66^{\mathrm{a}}$ & $2,50^{\mathrm{ab}}$ & $2,32^{\mathrm{b}}$ & $2,27^{\mathrm{b}}$ \\
$29-42$ jours & $3,06^{\mathrm{a}}$ & $3,02^{\mathrm{a}}$ & $3,08^{\mathrm{a}}$ & $3,75^{\mathrm{b}}$ \\
$0-28$ jours & $2,30^{\mathrm{a}}$ & $2,21^{\mathrm{a}}$ & $2,06^{\mathrm{b}}$ & $2,01^{\mathrm{b}}$ \\
$0-42$ jours & $2,62^{\mathrm{ab}}$ & $2,53^{\mathrm{a}}$ & $2,49^{\mathrm{a}}$ & $2,73^{\mathrm{b}}$ \\
\hline
\end{tabular}

des deux premières semaines le taux de GCDSG n'a pas eu d'effet significatif sur l'ingestion alimentaire et l'indice de consommation $(P>0,05)$, on observe en seconde période (15-28 j) que les animaux recevant le régime à 20 p. 100 ingèrent moins d'aliment et ont un meilleur indice de consommation que ceux qui consomment la ration témoin $(P<0,01)$. Cet avantage est toutefois compensé au cours de la période suivante (29-42 j) par une consommation d'aliment et un indice de consommation plus élevés $(P<0,05)$.

Sur l'ensemble de la période expérimentale (0-42 j), l'incorporation de GCDSG n'a pas eu d'effet significatif sur la quantité d'aliment ingérée $(P>0,05)$. Le régime en contenant 20 p. 100 a cependant donné un indice de consommation plus élevé que ceux à 10 ou 15 p. 100 .

Les taux de mortalité observés sont de $5,2 \mathrm{p}$. 100 pour les régimes à 0 et 20 p. 100 et de 1,3 et 3,9 p. 100 , respectivement, pour ceux avec 10 et 15 p. 100 de GCDSG. Les différences entre traitements ne sont pas significa- 
tives $(P>0,05)$. Le nombre de poulets présentant une malformation des pattes tend à augmenter avec le taux d'incorporation de GCDSG, passant de 3,3 p. 100 pour l'aliment témoin à $4,6,6,6$ et 7,1 p. 100 , respectivement, pour les aliments contenant 10,15 et 20 p. 100 de GCDSG. Toutefois, les différences entre traitements ne sont pas significatives $(P>0,05)$.

\section{Évaluation économique de l'utilisation de GCDSG}

Sur la base d'un prix de la GCDSG de 42,6 francs $\mathrm{CFA} / \mathrm{kg}$, on observe que l'augmentation du taux de graines dans la ration, en substitution au maïs et à la farine de poisson, entraîne une diminution du coût de l'aliment (tabl. I et II). Le calcul de la marge brute a été effectué en supposant que les animaux présentant des malformations des pattes sont systématiquement écartés de la commercialisation, qui s'effectue sur pied. Sur cette base, on observe que !'utilisation d'aliment contenant 10 à $20 \mathrm{p}$. 100 de GCDSG permet de diminuer le coût alimentaire ; cependant, seuls les aliments en contenant 10 ou $15 \mathrm{p} .100$ ont permis d'augmenter la marge brute par poussin démarré, par rapport à l'aliment témoin (tabl. V).

TABLEAUV Évaluation économique.

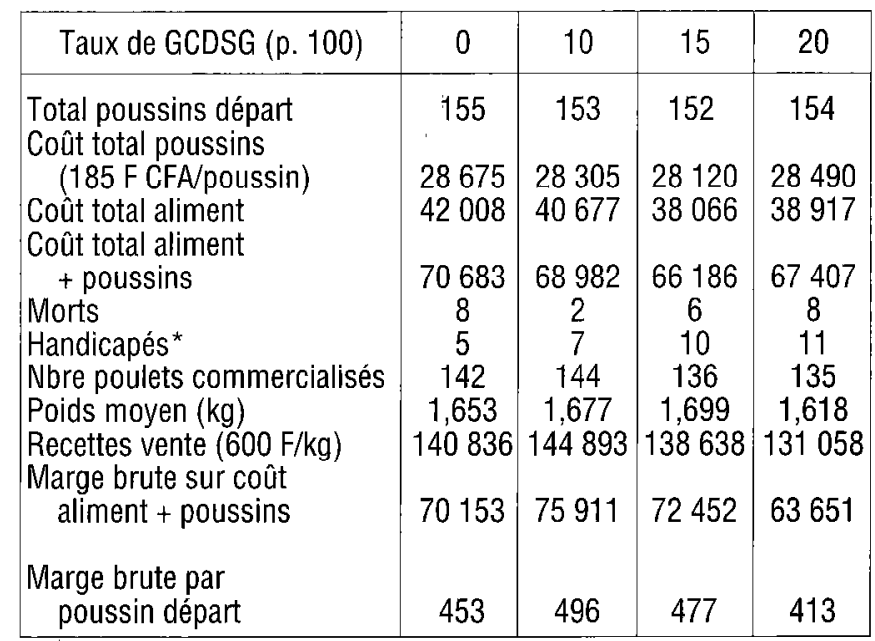

* Poulets présentant une malformation quelconque des pattes.

\section{DISCUSSION}

Les performances pondérales acceptables réalisées au cours de cette étude avec des rations contenant des taux élevés de GCDSG crues montrent que ce produit est bien valorisé par les poulets de chair. Ces résultats confirment ceux obtenus chez les porcs par LARUE et al. (15) et suggèrent que les mauvaises performances observées par les autres auteurs avec des graines de variétés classiques $(6,7,9,17)$ pourraient être dues à la présence de gossypol dans ces graines.

L'inclusion, dans les formules alimentaires, de GCDSG a entraîné une augmentation du taux de matières grasses (MG) atteignant 12 p. 100 dans les régimes à 20 p. 100 de graines. Les performances réalisées montrent qu'en milieu tropical des taux élevés de MG peuvent être supportés sans inconvénients majeurs. Sur l'ensemble de la période expérimentale, la consommation alimentaire et le gain de poids vif n'ont pas été significativement affectés par la présence de GCDSG dans l'aliment. Cependant, au cours de la période 15-28 j, on a observé un effet significativement favorable de la GCDSG sur l'indice de consommation. Ceci pourrait être attribué à un effet extracalorique de la graisse contenue dans la graine, ou à une sous-évaluation de la valeur énergétique réelle de ces régimes. En effet, certains auteurs ont observé que l'incorporation d'huile, d'origine végétale ou animale, dans des rations isoénergétiques permettait d'améliorer le gain de poids et l'indice de consommation (12) par un "effet extracalorique" de l'huile sur l'utilisation des nutriments de la ration $(8,21)$. Cependant, cet effet favorable de la GCDSG n'a pu être mis en évidence au cours de la période suivante (29-42 j) pour les régimes à 10 ou 15 p. 100 de GCDSG. Pour la ration à 20 p. 100 , on a noté, au cours de cette période, une ingestion alimentaire plus élevée et une diminution significative de l'efficacité de conversion de l'aliment. Ce résultat, quelque peu hétérogène et inattendu, semble indiquer que les niveaux énergétiques effectifs des rations alimentaires utilisées au cours de cette période (29-42 j) n'étaient pas identiques, ce qui pourrait provenir d'une erreur de fabrication, de formulation ou d'évaluation des matières premières.

On a observé que la fréquence des malformations de pattes tend à s'accroître $(P>0,05)$ avec l'augmentation du taux de GCDSG. Bien que non significative, cette augmentation semble suggérer que l'incorporation de GCDSG dans l'aliment réduit l'assimilation des éléments minéraux et ce d'autant plus que le taux d'incorporation est élevé. KENSETT et al. (12) ont observé que l'augmentation du taux de MG dans la ration entraîne une diminution de la fixation du phosphore et du calcium dans le tibia des poulets âgés de quatre semaines. De même, GRIFFITH et al. (10) ont montré que la digestibilité du calcium diminuait fortement avec un aliment contenant un taux élevé de MG.

Ces différents résultats suggèrent qu'une étude approfondie doit être menée afin de mieux connaître la valeur nutritive effective de la GCDSG, et son effet à des taux élevés dans des régimes alimentaires proposés à différentes phases de croissance des poulets de chair. 
L'étude économique succincte réalisée sur la base du prix de vente des graines de coton (23 francs CFA/kg) actuellement pratiqué par les usines d'égrenage de la Compagnie Ivoirienne pour le Développement des Textiles (CIDT) permet de penser que la graine de coton pourrait être valorisée de manière rentable dans l'alimentation animale. Toutefois, l'intérêt macro-économique d'une telle utilisation est fonction des possibilités alternatives de valorisation, notamment dans les huileries.

\section{CONCLUSION}

Dans l'ensemble, les résultats obtenus montrent que la graine de coton décortiquée issue de variétés sans gossypol pourrait constituer une nouvelle ressource alimentaire de bonne valeur nutritive pour les monogastriques.

YO (T.). Use of dehulled glandless cottonseed (DGCS) in broiler diets in Côte-d'Ivoire. Revue Élev. Méd. vét. Pays trop., 1991, 44 (3) : 355-360

The use of dehulled glandless cottonseed (DGCS) as protein and energy sources in broiler diets was tested comparing four diets with either $0,10,15$ or $20 \%$ DGCS. No significant effect on the live weight gain in the broilers was noted at $14,28,42$ days of age. For the whole experimental period (0-42 days), the feed intake was not affected by the level of DGCS in the feed. However, the feed conversion ratio was higher for the diet with $20 \%$ compared with those containing 10 or $15 \%$. Mortality rate was not affected but the number of birds with leg abnormalities tended to increase with DGCS level in the diet. For a DGSC price of $42.6 \mathrm{~F} \mathrm{CFA} / \mathrm{kg}$, its inclusion in the diet caused a decrease in feed costs and an increase in the gross margin per animal for chicks fed 10 or $15 \%$ DGCS. This product may constitute a new feed ingredient that can be advantageous in poultry feeding. Kev words : Broiler chicken - Feeding - Cottonseed - Feed efficiency - Live weight gain - Côte-d'Ivoire.
Son utilisation offre la possibilité de formuler des rations hautement énergétiques. La vulgarisation actuelle des variétés glandless devrait permettre, à terme, de disposer d'importantes quantités de graines dont une partie plus ou moins importante pourrait être valorisée dans l'alimentation animale. L'intérêt économique de son utilisation pour les volailles sera fonction de son prix de cession.

\section{REMERCIEMENTS}

L'auteur tient à remercier les responsables du département des cultures industrielles de l'IDESSA et particulièrement M. Yougo OURAGA pour avoir bien voulu mettre à notre disposition la matière première utilisée au cours de cette étude.

YO (T.). Utilización directa de semillas de algodón descascarilladas de variedades sin gosipol en la alimentación de pollos de engorde en Costa de Marfil. Revue Élev. Méd. vét. Pays trop., 1991, 44 (3) : 355-360

Se comprobó la utilización de semillas de algodón descascarilladas sin gosipol (SADSG) como fuente de proteinas y de energía en la alimentación de pollos de engorde al comparar cuatro raciones conteniendo $0,10,15$ 0 20 p. 100 de SADSG. No se observó ningún efecto significativo sobre el aumento de peso vivo de los animales a 14,28 y 42 días de edad. Durante los 42 días de la experimentación el porcentaje de SADSG en el pienso no tuvo efecto significativo sobre la cantidad ingerida. Sin embargo, con 20 p. 100 de SADSG, se obtuvo un indice de consumo superior al notado con 10 o 15 p. 100 . La tasa de mortalidad no varió, sino se observó una tendencia a la aumentación de la frecuencia de malformaciones de las patas. A partir de un precio teórico de las SADSG de 42,6 francos $\mathrm{CFA} / \mathrm{kg}$, la incorporación en la ración disminuye el coste alimenticio y aumenta la ganancia bruta por animal para los piensos conteniendo 10 o 15 p 100 de SADSG. Estas podrían constituir un nuevo recurso interesante para la alimentación de las aves de corral. Palahras claves: Pollo de engorde Alimentación - Semilla de algodón - Eficiencia alimenticia - Aumento de peso - Costa de Marfil

\section{BIBLIOGRAPHIE}

1 ANCEY (G.), LETENNEUR (L.). Étude de l'utilisation rationnelle des sous-produits agro-industriels par l'élevage et l'aquaculture. Paris, SEDES, Maisons-Alfort, IEMVT, 1989. 466 p.

2. Association of Official Analytical Chemists (AOAC). Official methods of analysis. 12 th ed. Washington, AOAC.

3. BESANCON (P), HENRY (O), ROUANET (J.M.). Valeur nutritionnelle comparée des farines délipidées de coton glandless et de soja. In : Le cotonnier sans gossypol, une nouvelle ressource alimentaire. Actes du colloque IDESSA-CIDT-TRITURAF, Abidjan, Côte-d'Ivoire, 26-27 novembre 1985. P. 97-110.

4. CALVET (H.). Graine et tourteau de coton en embouche intensive. In : Embouche intensive des bovins en pays tropicaux. Actes du colloque, Dakar, Sénégal, 4-8 décembre 1973. Maisons-Alfort, IEMVT, 1974. P. 63-68.

5. CAMPBELL (L.D.). Canola meal as a substitute for cottonseed meal in the diet of broiler chickens. Nutr. Rep. Int., $1988,37: 371$ 377. 
6. CLAWSON (A.J.), MANER (J.H.), GOMEZ (G.), MEJIA (O.), FLORES (Z.), BUITRAGO (J.). Unextracted cottonseed in diets for monogastric animals. 1. The effect of ferrous sulfate and calcium hydroxide in reducing gossypol toxicity. J. Anim. Sci., 1975, $40: 640647$.

7. CLAWSON (A.J.), MANER (J.H.), GOMEZ (G.), MEJIA (O.), FLORES (Z.), BUITRAGO (J.). Unextracted cottonseed in diets for monogastric animals. 2. The effect of boiling and oven $v s$ sun drying following pretreatment with a ferrous sulfate solution. $J$. Anim. Sci., 1975, 40 :648-654.

8. DAGHIR (N.J.). Nutrients requirements of laying hens under high temperature conditions. In : Poultry production in hot climates. 2nd DLG Symposium, Goslar, Germany, June 16-18, 1985 P. 81-98.

9. FITZSIMMONS (R.C.), NEWCOMBE (M.), MOUL (I.E.). The long-term effects of feeding ground and whole cottonseed to laying hens. Cun. J. Anim. Sci., 1989, $69: 425-429$.

10. GRIFFITH (F.D.), GRAINGER (R.B.), BEGIR (J.J.). The effect of dietary fat and cellulose on apparent calcium digestibility in growing chickens. Poult. Sci., 1961, $40: 1492-1497$.

11. INRA. L'alimentation des animaux monogastriques : porc, lapin, volaille. Paris, INRA, 1984. 282 p.

12. KENSETT (B.C.), HO (S.K.), TOUCHBURN (S.P.). Influence of level of dietary fat on the growth of dwarf chickens. Poult. Sci., $1980,59: 2065-2070$.

13. KOUAKOU (B.). Élude comparative du tourteau de soja et du tourteau de coton sans gossypol sur les performances des poulets de chair. Mém. fin études agron., Abidjan, ENSA, 1987. 48 p.

14. KUTCHES (A.J.), CHALUPA (W.), TREI (J.). Delinted cottonseed improves lactation responses. Feedstuffs, $1987,17: 16-17$.

15. LARUE (C.), KNABE (D.A.), IZARD (R.S.), TANKSLEY (T.D.). Glandless cottonseed kernels for starter, grower and finisher swine. J. Anim. Sci., 1987, 64: 1051-1059.

16. MOHAMED (K.), LARBIER (M.), LECLERCQ (B.). A comparative study of the digestibility of soyabean and cottonseed meal amino acids in domestic chicks and muscovy ducklings. Annls Zootech., 1986, 35 : 79-85.

17. NESER (J.A.), GRIMBECK (P.J.), LANGFORD (M.J.), NEL (P.W.). Gossypol poisoning in pigs. J. S. Afr. vet. Ass., 1988, 59 : 104.

18. NJIKE (N.C.). Cottonseed meal as substitute for groundnut cake in the diet of baby chicks. E. Afr. agric. For. J., 1975,44 : $59-69$.

19. NZEKWE (N.M.), OLOMU (J.M.). Cottonseed as substitute for groundnut meal in the ration of laying chickens and growing turkeys. J. Anim. Prod. Res., 1984, $4: 57-71$.

20. RYAN (J.R.), KRATZER (F.H.), GRAU (C.R.), VOHRA (P.). Glandless cottonseed meal for laying and breeding hens and broiler chicks. Poult. Sci., 1986, 65:949-955.

21. SCOTT (M.L.), NESHEIM (M.C.), YOUNG (R.J.). Nutrition of the chicken. 2nd ed. Ithaca, New York, Scott and Associates Publishers, 1976. 555 p.

22. SIBBALD (I.R.). The effect of dietary cellulose and sand on combined metabolic plus endogene energy and amino acid outputs of adult cockerels. Poult. Sci, 1980, 59:836-844

23. WALDROUP (P.W.). Cottonseed meal in poultry diets. Feedstuffs, 1981, 53 : 21-24.

24. YO ('l'). Low gossypol cottonseed meal as a substitute for soybean meal in broiler diets. Bouaké, IDESSA-CE, 1990. 9 p. Agribiologicae Research, 1991, 44 (à paraître).

25. YO (T.), BOUCIIEL (D.), KOUAO BROU (J.). Effets de différents niveaux de complémentation d'un fourrage vert (Panicum maximum) par la graine de coton mélassée sur la croissance de taurillons Baoulé en post-sevrage. Revue Élev. Méd. vét. Pays trop., $1990,43(4): 529-534$. 